\title{
Probability Distribution Analysis of Single-Molecule Fluorescence Anisotropy and Resonance Energy Transfer
}

\author{
Stanislav Kalinin*, Suren Felekyan, Matthew Antonik*, Claus A. M. Seidel* \\ Institut für Physikalische Chemie, Lehrstuhl für Molekulare Physikalische Chemie, Heinrich- \\ Heine-Universität, Universitätsstraße 1, Geb 26.32, 40225 Düsseldorf, Germany
}

Section 1: Effect of background subtraction. Background subtraction is not always sufficient even when high threshold values are applied. For instance, the data shown in Fig. 1B corrected by simple background subtraction cannot be satisfactory fitted, yielding $\chi_{r}^{2}=31.8$ for a low threshold $S_{\min }=5$ (Fig. S1A) and $\chi_{r}^{2}=3.34$ for a high threshold $S_{\min }=20$ (Fig. S1B).
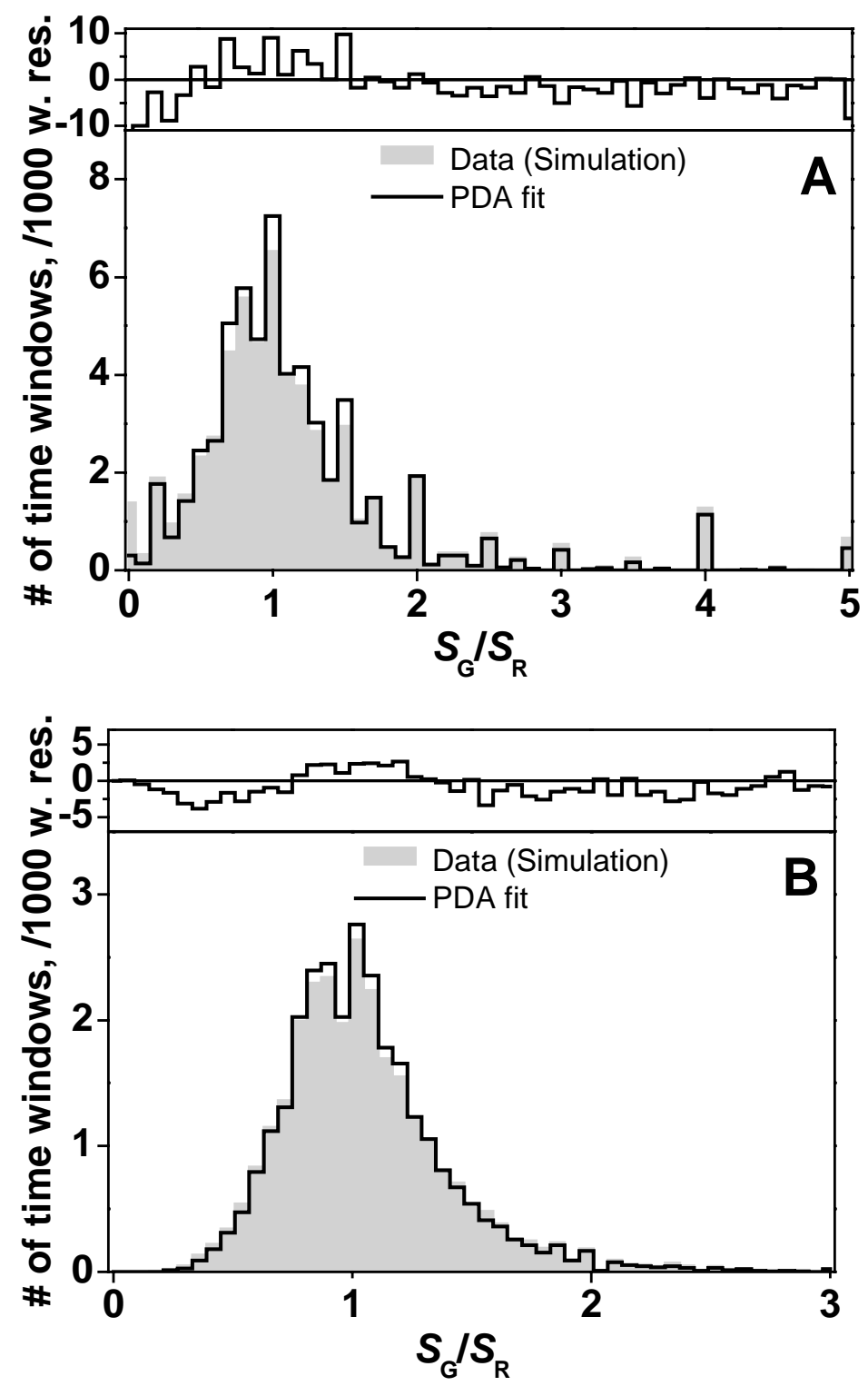

Figure S1. Analysis of simulated $S_{\mathrm{G}} / S_{\mathrm{R}}$ signal ratio histograms, corrected by background subtraction. The data set and simulation parameters are the same as shown in Fig. 1. A: Threshold $S_{\min }=5, S_{\max }=200 ; \mathbf{B}$ : Threshold $S_{\min }=20, S_{\max }=200$. 
Section 2: Comparison of ratiometric and normalized functions displaying the same data set
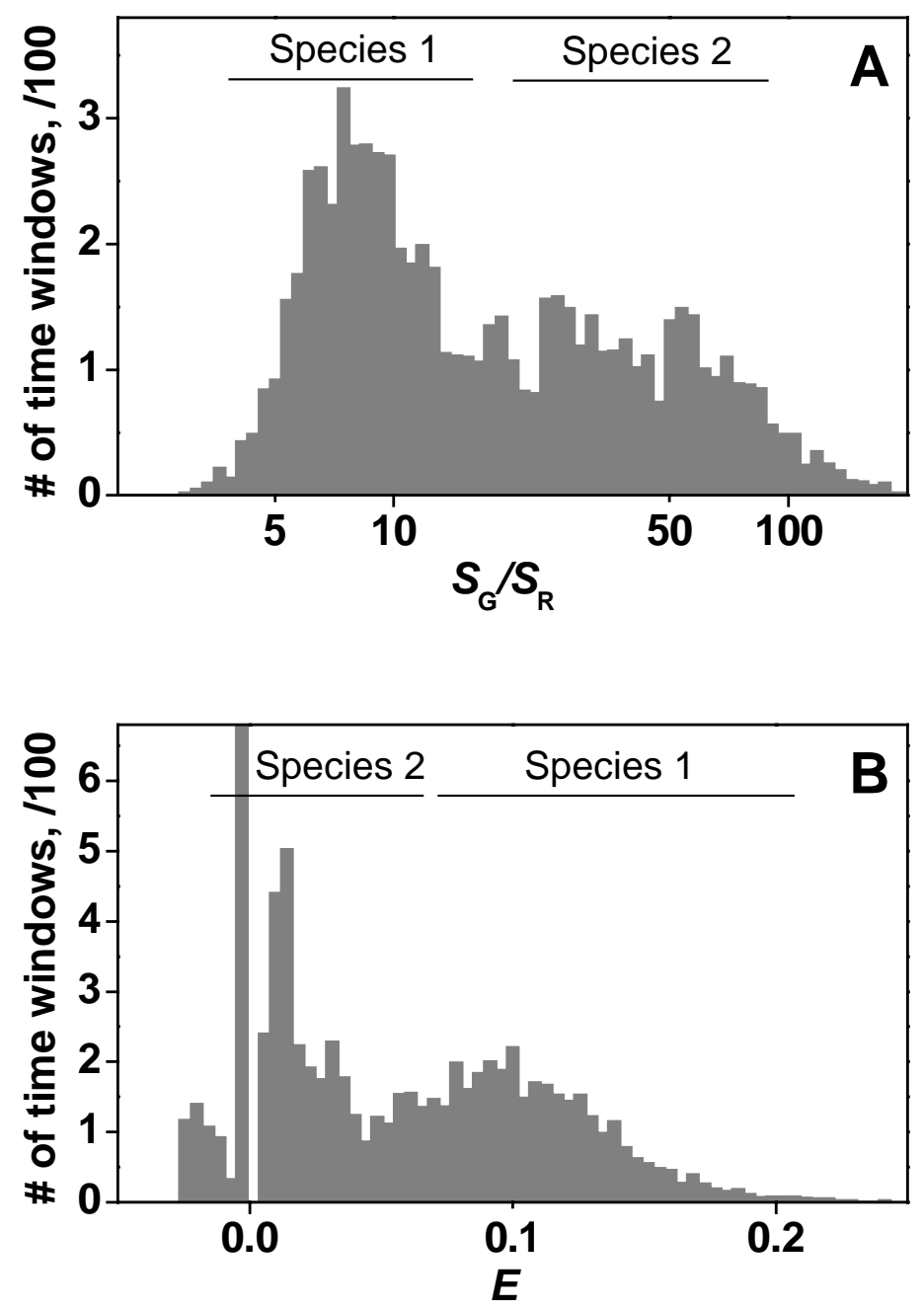

Figure S2. 1D histograms of $S_{\mathrm{G}} / S_{\mathrm{R}}$ signal ratio (A) and FRET efficiency (eq 16, B) generated from the same data set. A mixture of two states is simulated with the following parameters: "Species 1" $E_{1}=0.1(50 \%)$; "Species 2" $E_{2}=0.01(50 \%) ;\left\langle B_{\mathrm{G}}\right\rangle=2 ;\left\langle B_{\mathrm{R}}\right\rangle=1.2$. The Edistribution of "species 2 " is severely distorted. 